\title{
Fatty acid desaturase 2 (FADS2) insertion/deletion polymorphism impact on muscle fatty acid profile in European grayling (Thymallus thymallus)
}

\author{
Bénédicte Renaville*, Francesca Tulli, Massimiliano Bruno, Emilio Tibaldi and Maria Messina \\ Department of Food Science, University of Udine, Via Sondrio 2A, 33100 Udine, Italy
}

(Submitted 14 September 2012 - Final revision received 18 January 2013 - Accepted 6 March 2013 - First published online 12 April 2013 )

\section{Abstract}

An insertion (In)/deletion (Del) polymorphism in the fatty acid desaturase 2 (FADS2) gene, which codes for $\triangle 6$-desaturase, was for the first time observed in fish. The polymorphism is located in the seventh intron of FADS2 and the insertion consists of eleven repeats of CTGT ( $44 \mathrm{bp)}$ with an allelic frequency, for the insertion, of $39 \%$. The polymorphism was associated with a modulation in $\Delta 6$-desaturase activity as significant effects on the ratio of EPA or DHA to their precursors were found $(P<0 \cdot 001)$. A different distribution of SFA, MUFA and PUFA among the In/In, In/Del and Del/Del groups was also detected in fish fillet. The results suggest that genetic selection for this marker might improve the ability of European grayling to utilise dietary $n$-3 long-chain PUFA precursors, as $\Delta 6$-desaturase is the rate-limiting enzyme in the production of EPA and DHA from $\alpha$-linolenic acid.

\section{Key words: $\Delta 6$-Desaturase: EPA: DHA: Thymallidae: Mutations: Fish}

Fish have the ability to elongate and/or desaturate fatty acid precursors to synthesise long-chain (LC) PUFA ${ }^{(1)}$, but the efficiency of this metabolic pathway varies among fish species. In contrast to marine finfish species where the ability of synthesising LC-PUFA from precursors is limited and controversial $^{(2,3)}$, freshwater fish seem to be able to maintain a certain degree of $\Delta 6-, \Delta 5$-desaturase and elongase activities to produce arachidonic acid, EPA and DHA if their precursors linoleic and $\alpha$-linolenic acids (ALA) are present in their diet. Fatty acid desaturase 2 (FADS2), also called $\Delta 6$-desaturase, is the rate-limiting enzyme in the production of EPA and DHA from $\operatorname{ALA}^{(4)}$ even if two other enzymes (FADS1/D5-desaturase and elongase) are involved in this biosynthetic pathway. FADS2 is a membrane-bound protein with amino-terminal cytochrome $b 5$ domains carrying haem-binding motifs, twomembrane-spanning domains and three His-box motifs ${ }^{(5)}$. FADS2 converts ALA $(18: 3 n-3)$ into stearidonic acid $(18: 4 n-3)$ and linoleic acid $(18: 2 n-6)$ into $\gamma$-linolenic acid $(18: 3 n-6)$, which are the first step in the pathway of $n-3$ and $n-6$ LC-PUFA synthesis, respectively.

$\Delta 6$ - and $\Delta 5$-Desaturases are of major interest in the field of fish nutrition, especially with regard to the possibility of dietary linoleic and ALA to fulfil the requirement of teleosts for the essential LC-PUFA of the $n-3$ and $n-6$ series. Until recently, LC-PUFA requirements have been satisfied by lipids from fishery-derived products, but such LC-PUFA sources have reached their limit of sustainability ${ }^{(6,7)}$. The ability to convert linoleic and $\alpha$-linolenic fatty acids, abundant in alternative lipid sources such as vegetable oils, into LC-PUFA is well established in some species, such as rainbow trout $^{(8-12)}$, but has been poorly investigated in other freshwater species, including European grayling ${ }^{(13)}$. Individual variability in the capacity of fish to retain or synthesise $n-3$ LC-PUFA when fed vegetable oil diets has been shown in Atlantic salmon ${ }^{(14)}$. Moreover, Leaver et al. ${ }^{(15)}$ demonstrated that flesh $n$-3 LC-PUFA composition is a highly heritable trait $\left(b^{2}=0.77\right)$ in salmon. However, in their study, Morais et al. ${ }^{(16)}$ showed that the hepatic expression of $\Delta 6$-desaturase was not responsible for the variations observed in the levels of $n-3$ LC-PUFA in four families of Atlantic salmon. Therefore, these authors have suggested that the observed variability might originate from the presence of different alleles of fatty acyl desaturases encoding proteins with altered biological activity or specificity.

Recent findings have outlined that genetic variation in FADS1 and FADS2 gene clusters is associated with altered desaturase activity in human subjects ${ }^{(17)}$. Moreover, FADS2 polymorphism has been shown to modulate the activity of $\Delta 6$-desaturase in human subjects ${ }^{(18)}$, Japanese quails ${ }^{(19)}$ and pigs ${ }^{(20)}$; however, the association with desaturase activity has not yet been examined in fish. Therefore, FADS2 is a candidate gene for increasing EPA and DHA synthesis from ALA in fish.

Abbreviations: ALA, $\alpha$-linolenic acid; FADS2, fatty acid desaturase 2; LC-PUFA, long-chain PUFA.

*Corresponding author: B. Renaville, fax +39432 558130, email benedicte.renaville@uniud.it 
Thus, the present study aimed to study the $\Delta 6$-desaturase polymorphism and its impact on the muscle fatty acid profile in European grayling (Thymallus thymallus L., 1758), chosen as a cold freshwater fish model. This fish species has a natural distribution covering vast parts of Europe, from France and England eastwards to the Ural Mountains and from Scandinavia southwards to the Balkan region and Northern Italy ${ }^{(21)}$. Actually, there is a growing interest for this species in fisheries management and conservation plans (European Dir. Habitat 92/43/CEE) and, recently, also in aquaculture.

\section{Materials and methods}

\section{Animal and rearing conditions}

For the present study, 100 specimens of European grayling (initial body weight $12 \cdot 1$ (SD $0 \cdot 1) \mathrm{g}$ ) were obtained from the hatchery Ribiška Druzina (Tolmin, Slovenija). Fish originated from a single stock were obtained by mating one 4-year-old female and two males. They were stocked in a 500-litre fibreglass tank in the experimental flowthrough facilities of the Department of Food Science at the University of Udine (Italy) supplied with well water (water temperature $13.7 \pm 0.22^{\circ} \mathrm{C}$; dissolved oxygen $8.17(\mathrm{sD} 0.8) \mathrm{mg} / \mathrm{l} ; \mathrm{pH}$ $7 \cdot 49 \pm 0.21$; total ammonia nitrogen 0.08 (SD 0.01) mg/l; photoperiod $12 \mathrm{~h}$ light $-12 \mathrm{~h}$ dark). After stocking, fish were hand-fed a commercial diet for trout (feed formula: $42 \%$ crude protein, $25 \%$ crude lipid on dry feed, Dibaq ATX 5HMDI; Dibaq Italia) in two daily meals (09.00 and 16.00 hours) until the first feed item was refused, over $64 \mathrm{~d}$. At the end of the trial, fish were weighed (final weight 31.8 (SD 2.5) g) under moderate anaesthesia ( $5 \mathrm{mg}$ benzocaine/ 1 alcohol solution) and twenty-seven specimens were killed with excess of the anaesthetic $(30 \mathrm{mg}$ benzocaine/l alcohol solution) to obtain the fillet. Handling of the fish was done according to the guidelines of the European Union Directive (2010/63/EU) on the protection of animals used for scientific purposes.

\section{Molecular genetic marker evaluation}

DNA was extracted from muscle samples (twenty-seven animals) using a standard DNA extraction method (GenElute Mammalian Genomic DNA Miniprep Kit; Sigma-Aldrich). A primer set (forward: AAAGAAGTTGAAGTACATGCCCTA; reverse: AGTTCCGTTGTGAAAACATGG) was designed in order to amplify a fragment of the FADS2 gene based on the
Salmo salar sequence (GenBank accession no. AY736067.2). The forward primer was designed in $S$. salar exon 7 and the reverse one in exon 8 . PCR conditions consisted of an initial step at $94^{\circ} \mathrm{C}$ for $3 \mathrm{~min}$ followed by forty cycles of $94^{\circ} \mathrm{C}$ for $30 \mathrm{~s}$, annealing temperature $\left(60^{\circ} \mathrm{C}\right)$ for $30 \mathrm{~s}$ and $72^{\circ} \mathrm{C}$ for $30 \mathrm{~s}$. The final extension step consisted of $5 \mathrm{~min}$ at $72^{\circ} \mathrm{C}$. PCR products were separated on a $2.5 \%$ agarose gel in $1 \times$ TBE buffer by electrophoresis. Separated fragments on agarose gels were sized by referencing with a $100 \mathrm{bp}$ DNA ladder (Fig. 1). The bands were cut and DNA extracted with a gel extraction kit (Zymoclean Gel DNA Recovery Kit; Zymo Research). PCR products were sequenced from the gel extract using an ABI automated DNA sequencer (Applied Biosystems).

\section{Sequence comparison}

The sequence of European grayling (T. thymallus) was compared with the sequence of Atlantic salmon ( $S$. salar, GenBank accession no. AY736067.2), cod (Gadus morbua, Ensembl ENSGMOG00000017746) and zebrafish (Danio rerio, Ensembl ENSDARG00000019532) using BLAST (bl2seq) from NCBI.

\section{Lipid analysis}

Muscle lipids were extracted with a chloroform-methanol $(2: 1, \mathrm{v} / \mathrm{v})$ solution containing $0.01 \%$ butylated hydroxytoluene according to Folch et al. $^{(22)}$.

A $5 \%$ solution of $\mathrm{HCl}$ in methanol was used to prepare fatty acid methyl esters according to Christie ${ }^{(23)}$. Fatty acid methyl esters were separated and quantified by high-resolution GC (TRACE GC2000; Fisher Scientific SAS) using a $30 \mathrm{~m} \times 0.32 \mathrm{~mm}$ (inner diameter) Omegawax ${ }^{\mathrm{TM}}$ Capillary GC Column (Supelco) with flame ionisation detection at $250^{\circ} \mathrm{C}$. The carrier gas $\left(\mathrm{H}_{2}\right)$ flow rate was $1 \mathrm{ml} / \mathrm{min}$, and samples $(1 \mu \mathrm{l})$ were directly injected in a split ratio of 1:30. The oven temperature was programmed from $180^{\circ} \mathrm{C}$ for $6 \mathrm{~min}$, then at $3^{\circ} \mathrm{C} / \mathrm{min}$ until $225^{\circ} \mathrm{C}$ and held for $10 \mathrm{~min}$. The chromatograms were acquired and processed using ChromQuest integration software (ThermoQuest Italia Limited) and individual methyl esters were identified by comparison with known standards obtained from Supelco, Inc. Repeated injections of the standard solution were carried out to test the analytical precision. All solvents and reagents were of analytical grade and were purchased from Sigma-Aldrich, Inc. Data on individual

$\begin{array}{llllllllllllllllll}1 & 2 & 3 & 4 & 5 & 6 & 7 & 8 & 9 & 10 & 11 & 12 & 13 & 14 & 15 & 16 & 17 & 18\end{array}$

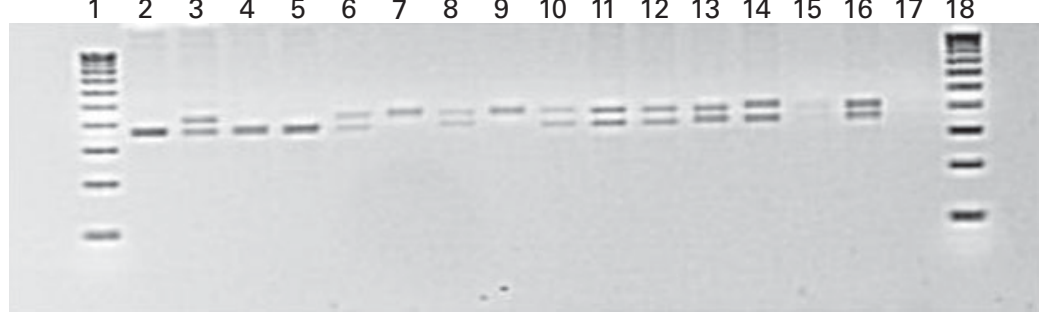

Fig. 1. PCR products of fatty acid desaturase 2. First and last lanes: molecular weight ( $100 \mathrm{bp} / \mathrm{band})$; second, fourth and fifth lanes: homozygous for the deletion; seventh and ninth lanes: homozygous for the insertion; other lanes: heterozygous. 
fatty acid composition are expressed as a percentage of total identified fatty acids.

\section{Data analysis}

Insertion/deletion associations with fatty acid proportions were analysed using the general linear mixed model procedure of SPSS version 17 (SPSS, Inc.) with a model that included insertion/deletion as a fixed effect. Significant differences were declared when the marker genotype effect was a significant source of variation in ANOVA, and the $P$ value for the difference between the least-squares means for each marker genotype was less than 0.05. If appropriate, the post boc least significant difference test was applied to compare the means.

\section{Results}

Sequencing revealed an insertion/deletion polymorphism in the seventh intron of FADS2. The insertion consists of eleven repeats of CTGT ( $44 \mathrm{bp}$ ); indeed, in this intronic region, the motif CTGT is repeated either forty times (insertion allele) or twenty-nine times (deletion allele). The allelic frequency was 39\% for the insertion (obtained by adding half of the heterozygous frequency to the homozygous frequency) and $61 \%$ for the deletion.

The sequence for the deletion variant of the amplified fragment of FADS2 of European grayling is reported in Fig. 2 along with Atlantic salmon ( $S$. salar), cod ( G. morbua) and zebrafish (D. rerio) sequences. With respect to the exons, the intronic region was more dissimilar between salmon and grayling and the repeated motif was not present in $S$. salar. The grayling sequence of the exonic region was quite similar to that of $D$. rerio and $G$. morbua, while the intronic region was completely dissimilar.

The total lipid content of the fish fillets and the fatty acid composition associated with the observed polymorphism are presented in Table 1.

The FADS2 polymorphism is associated with clear differences in the incidence of specific fatty acid ratio along the desaturation pathway that almost reached significance in the case of $18: 4 n-3$ to $18: 3 n-3(P=0 \cdot 088)$, but resulted highly significant in the case of $20: 5 n-3$ to $18: 3 n-3(P=0 \cdot 001)$ and $22: 6 n-3$ to $18: 3 n-3(P=0 \cdot 001)$. Such a clear effect of the polymorphism was not observed in the ratio of $n-6$ fatty acids, although the activity of $\Delta 6$-desaturase seemed to be lower in the Insertion/Insertion fish. The mutation is also associated with significant differences in palmitoleic acid, ALA, stearidonic acid and DHA percentages as well as SFA and MUFA.

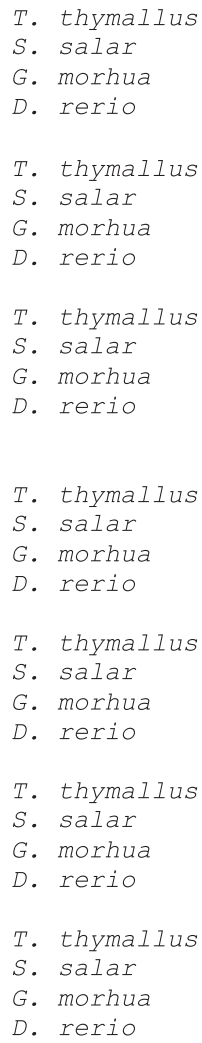

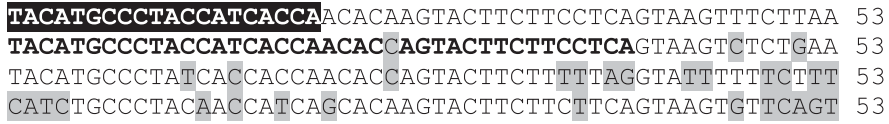

CAAGTTTCATGAATTTCTGTAAAG-----CATCAACTTCCATTGAACACAT 99 CAAGTTTCATGGATTTCTGTATATAAATCACATCAACTTCCATTGAACACAT 105 GATTCATTTTTTATATTTTCTTAGAAGTAATTGAAA--TCCATTGAATTGCC 103 GTTAGGGATTCCTACTGAAATGTTTATTATAATGGTAACACTTTACAATAAG 105

AAAAGCAAAAGATAGACGTCTGTCTGTCTGTCTGTCTGTCTGTCTGTCTGTC 151

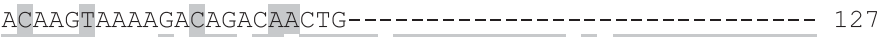
ACTTAAAAAAAAAGCATGTTGAATTGCCCGAAACAATTTGAACTGTCGGTGC 155 GTTCATTAGTTAATGCATTTACTAACCTGAACTAATCATGAACAACACATTT 157

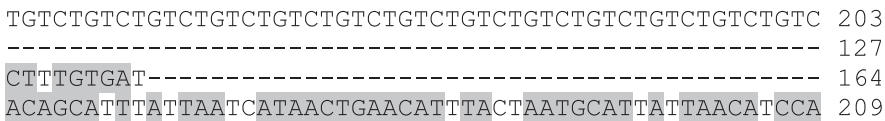

TGTCTGTCTGTCTGTCTGTCTGTCTGTCTGAATGAATGAATGAAATACCAAT 255 --------------------------------------------CTTTC 169 AgtcCAtgcttgtTAACATtAgtTAAtgCACCATGAgtTAACA[.. ]ACtgA 263

GTCGCATTTTCCTTCTGTCTCTCAGTTGGACCTCCACTACTCATTCCAGTGT 307 CCAATATTTTCCTTCTGTCTTTCAGTTGGACCTCCACTACTTATTCCAGTGT 195 ATGAGTATGTCTTTGGCTCCTTCAGTCGGACCCCCACTGCTCATCCCGGTGT 221 TGTTTCCTCTTTTATTTCCCCCCAGTTGGTCCTCCCCTGCTCATCCCAGTGT 315

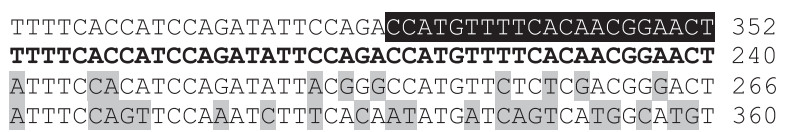

Fig. 2. Comparison of the nucleotide sequence of fatty acid desaturase 2 (FADS2) from Thymallus thymallus (deletion variant) with that of FADS2 from Salmo salar, Gadus morhua and Danio rerio. Sequences were aligned using Blast (bl2seq from the NCBI). White letters, black background: primers used; bold: salmon exons; grey background: sequence differences from T. thymallus. 
Table 1. Genotypic least-squares means for fatty acid desaturase 2 on total lipid content $(\mathrm{g} / 100 \mathrm{~g}$ muscle) and fatty acid concentrations (\% total fatty acids)

(Least-squares mean values with their standard errors)

\begin{tabular}{|c|c|c|c|c|c|c|c|}
\hline & \multicolumn{2}{|c|}{$\ln / \ln$} & \multicolumn{2}{|c|}{ In/Del } & \multicolumn{2}{|c|}{ Del/Del } & \multirow[b]{2}{*}{$P$} \\
\hline & LSM & SE & LSM & SE & LSM & SE & \\
\hline \multicolumn{8}{|l|}{ Fatty acid percentage } \\
\hline $10: 0$ & $0 \cdot 13^{\mathrm{a}}$ & 0.11 & $0.40^{\mathrm{b}}$ & 0.05 & $0.55^{\mathrm{b}}$ & 0.07 & 0.03 \\
\hline $12: 0$ & $3.07^{\mathrm{a}}$ & 1.98 & $7 \cdot 83^{b}$ & 0.89 & $9.53^{\mathrm{b}}$ & $1 \cdot 15$ & 0.03 \\
\hline $14: 0$ & 3.33 & $1 \cdot 72$ & $5 \cdot 15$ & 0.77 & $6 \cdot 77$ & 1.00 & NS \\
\hline $16: 0$ & $14 \cdot 3$ & 0.75 & $14 \cdot 1$ & 0.34 & $13 \cdot 6$ & 0.43 & NS \\
\hline $16: 1$ & $3 \cdot 21^{a, b}$ & 0.67 & $2 \cdot 73^{\mathrm{a}}$ & 0.30 & $1.68^{b}$ & 0.39 & 0.065 \\
\hline $18: 0$ & 3.42 & 0.24 & 3.53 & $0 \cdot 11$ & $3 \cdot 77$ & 0.14 & NS \\
\hline $18: 1 n-9$ & $20 \cdot 8$ & $1 \cdot 70$ & $19 \cdot 4$ & 0.76 & $17 \cdot 8$ & 0.98 & NS \\
\hline $18: 1 n-7$ & $3 \cdot 12$ & 0.96 & $2 \cdot 83$ & 0.44 & 1.98 & 0.57 & NS \\
\hline $18: 2 n-6$ & 11.5 & 0.91 & $12 \cdot 7$ & 0.41 & $12 \cdot 3$ & 0.53 & NS \\
\hline $18: 3 n-6$ & 0.42 & 0.11 & 0.37 & 0.05 & 0.40 & 0.06 & NS \\
\hline $18: 3 n-3$ & $0.60^{\mathrm{a}}$ & 0.95 & $2 \cdot 19^{\mathrm{a}}$ & 0.43 & $3 \cdot 72^{b}$ & 0.55 & 0.02 \\
\hline $18: 4 n-3$ & $0.53^{\mathrm{a}}$ & $0 \cdot 18$ & $0.68^{a}$ & 0.08 & $1.08^{\mathrm{b}}$ & 0.11 & 0.01 \\
\hline $20: 0$ & 0.11 & 0.04 & 0.13 & 0.02 & 0.09 & 0.03 & NS \\
\hline $20: 1 n-9$ & 4.05 & 0.80 & $2 \cdot 98$ & 0.36 & $2 \cdot 13$ & 0.46 & NS \\
\hline $20: 4 n-6$ & $1 \cdot 11^{\mathrm{a}}$ & 0.11 & $0.85^{b}$ & 0.05 & $0.95^{\mathrm{a}, \mathrm{b}}$ & 0.06 & 0.08 \\
\hline $20: 4 n-3$ & 0.44 & 0.07 & 0.49 & 0.03 & 0.55 & 0.04 & NS \\
\hline $20: 5 n-3$ & 2.84 & 0.25 & 2.22 & 0.11 & 2.33 & 0.15 & NS \\
\hline $22: 6 n-3$ & $17 \cdot 2^{\mathrm{a}}$ & 1.43 & $13 \cdot 3^{b}$ & 0.64 & $13 \cdot 0^{\mathrm{b}}$ & 0.82 & 0.05 \\
\hline $18: 4 n-3$ to $18: 3 n-3$ & $0.87^{\mathrm{a}}$ & 0.18 & $0.48^{a, b}$ & 0.08 & $0.39^{b}$ & 0.10 & 0.088 \\
\hline $20: 5 n-3$ to $18: 3 n-3$ & $5 \cdot 27^{\mathrm{a}}$ & 0.87 & $1 \cdot 74^{\mathrm{b}}$ & 0.40 & $1.02^{b}$ & 0.50 & 0.001 \\
\hline $22: 6 n-3$ to $18: 3 n-3$ & $31 \cdot 1^{\mathrm{a}}$ & 4.84 & $10 \cdot 4^{\mathrm{b}}$ & $2 \cdot 24$ & $5 \cdot 6^{\mathrm{b}}$ & $2 \cdot 79$ & 0.001 \\
\hline $18: 3 n-6$ to $18: 2 n-6$ & 0.04 & 0.01 & 0.03 & 0.01 & 0.03 & 0.01 & NS \\
\hline $20: 4 n-6$ to $18: 2 n-6$ & $0 \cdot 10^{\mathrm{a}}$ & 0.01 & $0.07^{b}$ & 0.01 & $0.08^{a, b}$ & 0.09 & 0.080 \\
\hline SFA & $26 \cdot 4^{\mathrm{a}}$ & $3 \cdot 16$ & $32 \cdot 8^{a, b}$ & 1.42 & $35 \cdot 9^{\mathrm{b}}$ & 1.83 & 0.047 \\
\hline MUFA & $34.5^{\mathrm{a}}$ & $3 \cdot 15$ & $30 \cdot 4^{a, b}$ & 1.41 & $25 \cdot 7^{\mathrm{b}}$ & 1.82 & 0.043 \\
\hline$n-6$ PUFA & $14 \cdot 8$ & 1.01 & $16 \cdot 0$ & 0.45 & $15 \cdot 4$ & 0.59 & NS \\
\hline$n-3$ PUFA & 23.2 & 1.63 & $19 \cdot 0$ & 0.73 & $22 \cdot 2$ & 0.94 & NS \\
\hline Genotypic frequency (\%) & \multicolumn{2}{|c|}{11} & \multicolumn{2}{|c|}{56} & \multicolumn{2}{|c|}{33} & \\
\hline Fat content $(\mathrm{g} / 100 \mathrm{~g})$ & $2 \cdot 36$ & 0.32 & $2 \cdot 76$ & 0.14 & $2 \cdot 60$ & 0.19 & NS \\
\hline
\end{tabular}

In, insertion; Del, deletion.

a,b Mean values within a row with unlike superscript letters were significantly different $(P<0.05)$.

\section{Discussion}

In the present study, we revealed the presence of a polymorphism in the FADS2 gene of T. thymallus. The repeated motif where we observed the insertion/deletion polymorphism was not present in S. salar nor in G. morbua or in $D$. rerio sequences, suggesting that if these species present polymorphisms in the FADS2 gene, they will be different.

The effect of the FADS2 polymorphism on $\Delta 6$-desaturase activity was first observed in human studies ${ }^{(24)}$ where an association with the EPA:ALA ratio was also outlined. Moreover, in European adolescents, one minor allele of the FADS1-FADS2 gene cluster was associated with higher $\Delta 6$-desaturase activity levels ${ }^{(25)}$. More recently, FADS2 polymorphisms have also been observed in other mammals, such as pigs ${ }^{(20)}$, where the authors have also shown a modulation of $\Delta 6$-desaturase activity. The insertion/deletion polymorphism in the seventh intron of the FADS2 gene of T. thymallus observed in the present study affected DHA and ALA proportions in fillet lipids, which is in agreement with studies on human subjects showing that SNP in the FADS1-FADS2 gene cluster reduces EPA concentrations and increases ALA concentrations in plasma phospholipids ${ }^{(17,26)}$, but also in breast milk ${ }^{(27)}$ and adipose tissue ${ }^{(28)}$. Several other studies in human subjects have shown the associations between mutations in the FADS1-FADS2 gene cluster and fatty acid composition (reviewed in Merino et $\mathrm{al}^{\left({ }^{(18)}\right)}$. Consistently, Khang et $a l^{(19)}$ observed significant associations between the FADS2 polymorphism and the DHA content in the egg yolk of Japanese quail.

Also in the present study, the ratio of products:precursors was used to assess the activity of $\Delta 6$-desaturase, as already proposed by Merino et al. ${ }^{(17)}$. The insertion of the repeated motif resulted in an increase of $\Delta 6$-desaturase activity in fatty acid biosynthetic pathways for $n$-3 PUFA. However, the polymorphism does not seem to affect the activity of the enzyme on $n-6$ PUFA. This might result from a different activity of the enzyme on the different precursors ( $n-3$ or $n-6)$, as it has been observed in $\operatorname{dog} s^{(29)}$ and Murray $\operatorname{cod}^{(30)}$. Moreover, Thanuthong et al. ${ }^{(31)}$ showed that, in the trout, the $\Delta 6$-desaturase has a $3 \cdot 2$-fold higher affinity towards $18: 3 n-3$ over $18: 2 n-6$. Despite a relatively limited number of animals analysed in the present study, these preliminary results suggest that the genetic selection of European grayling for this marker might improve its ability to utilise dietary LC-PUFA precursors, as FADS2 is the rate-limiting enzyme in the production of EPA and DHA from ALA. 
As nutritionists have recommended the consumption of fish for its beneficial properties due to the high content in LC-PUFA, namely EPA and DHA, which have a variety of important physiological functions ${ }^{(32-36)}$, a similar approach applied to other finfish species of aquaculture interest may, by using marker-assisted selection, allow an improvement in the utilisation of dietary vegetable oils and meals, rich in $n-3$ precursors, and also provide human consumers a healthier fish product.

\section{Acknowledgements}

The present study was supported by Ente Tutela Pesca Regione Friuli Venezia Giulia (Italy). The authors are thankful to Aysum Adakli, Enrico Daniso and Massimo Lanzoni for their precious technical assistance. B. R. and M. M. developed the initial idea, designed the study and were in charge of the genetic determinations; F. T. was in charge of the lipid analysis; M. B. was responsible for the fish trial; E. T. supervised the study at all steps. All the authors critically revised the drafted manuscript. None of the authors has any financial or personal conflicts of interest.

\section{References}

1. Sargent JR, Tocher DR \& Bell JG (2002) The lipids. In Fish Nutrition, pp. 181-257 [J Halver and R Hardy, editors]. San Diego, CA: Academic Press/Elsevier.

2. Monroig O, Zheng X, Morais S, et al. (2010) Multiple genes for functional $\Delta 6$ fatty acyl desaturases (Fad) in Atlantic salmon (Salmo salar L.): gene and cDNA characterization, functional expression, tissue distribution and nutritional regulation. Biochim Biophys Acta 1801, 1072-1081.

3. Tocher DR \& Ghioni C (1999) Fatty acid metabolism in marine fish: low activity of fatty acyl delta 5 desaturation in gilthead sea bream (Sparus aurata) cells. Lipids 34 , 433-440.

4. Cook H (1996) Fatty acids desaturation and chain elongation in eukaryotes. In Biochemistry of Lipids, Lipoproteins and Membranes New Comprehensive Biochemistry, 31, pp. 129-152 [DVJ Vance, editor]. Amsterdam: Elsevier.

5. Cho HP, Nakamura MT \& Clarke SD (1999) Cloning, expression, and nutritional regulation of the mammalian Delta-6 desaturase. J Biol Chem 274, 471-477.

6. Pike I \& Barlow S (2003) Impact of fish farming on fish stocks. Fish Farmer 26, 14-16.

7. Shepherd C, Pike I \& Barlow S (2005) Sustainable feed resources of marine origin. International Conference of 'Aquaculture Europe: Lessons from the Past to Optimise the Future', Trondheim, Norway, 5-9 August 2005. In European Aquaculture Society Special Publication No. 35, pp. 59-66.

8. Owen J, Ardon J, Middleton C, et al. (1979) Elongation and desaturation of dietary fatty acids in turbot Scophthalmus maximus L. and rainbow trout, Salmo gairdnerii. Lipids 10, 528-531.

9. Kanazawa A, Teshima S \& Ono K (1979) Relationship between essential fatty acid requirements of aquatic animals and the capacity for bioconversion of linolenic acid to highly unsaturated fatty acids. Comp Biochem Physiol $B \quad 63$, 295-298.

10. Buzzi M, Henderson RJ \& Sargent JR (1996) The desaturation and elongation of linolenic acid and eicosapentaenoic acid by hepatocytes and liver microsomes from rainbow trout (Oncorhynchus mykiss) fed diets containing fish oil or olive oil. Biochim Biophys Acta 1299, 235-244.

11. Mourente $G$ \& Tocher DR (1998) The in vivo incorporation and metabolism of [1-C-14] linolenate (18:3n-3) in liver, brain and eyes of juveniles of rainbow trout Oncorbynchus mykiss L. and gilthead sea bream Sparus aurata L. Fish Physiol Biochem 18, 149-165.

12. Seiliez I, Panserat S, Kaushik S, et al. (2001) Cloning, tissue distribution and nutritional regulation of a Delta6-desaturase-like enzyme in rainbow trout. Comp Biochem Physiol B 130, 83-93.

13. Tulli F, Cardinaletti G, Messina M, et al. (2011) Growth performance and fatty acid metabolism in European grayling (Thymallus thymallus, L.) fed diets differing in $n-3$ and $n-6$ PUFA levels. It J Anim Sci 10, 57-58.

14. Schlechtriem C, Bron JE \& Tocher DR (2007) Inter-individual variation in total fatty acid compositions of flesh of Atlantic salmon smolts-fed diets containing fish oil or vegetable oil. Aquacult Res 38, 1045-1055.

15. Leaver MJ, Taggart JB, Villeneuve L, et al. (2011) Heritability and mechanisms of $n$ - 3 long chain polyunsaturated fatty acid deposition in the flesh of Atlantic salmon. Comp Biochem Physiol Part D Genomics Proteomics 6, 62-69.

16. Morais S, Taggart J, Guy D, et al. (2012) Hepatic transcriptome analysis of inter-family variability in flesh $n-3$ longchain polyunsaturated fatty acid content in Atlantic salmon. BMC Genomics 13, 410.

17. Merino DM, Johnston H, Clarke S, et al. (2011) Polymorphisms in FADS1 and FADS2 alter desaturase activity in young Caucasian and Asian adults. Mol Genet Metab 103, $171-178$

18. Merino D, Ma D \& Mutch D (2010) Genetic variation in lipid desaturases and its impact on the development of human disease. Lipids Health Dis 9, 63.

19. Khang NTK, Jennen DGJ, Tholen E, et al. (2007) Association of the FADS 2 gene with omega- 6 and omega-3 PUFA concentration in the egg yolk of Japanese quail. Anim Biotechnol 18, 189-201.

20. Renaville B, Prandi A, Fan B, et al. (2013) Candidate gene marker associations with fatty acid profiles in heavy pigs. Meat Sci 93, 495-500.

21. Northcote TG (1995) Comparative biology and management of Artic and European grayling (Salmonidae, Thymallus). Rev Fish Biol Fisheries 5, 141-194.

22. Folch J, Lees M \& Sloane Stanley GH (1957) A simple method for the isolation and purification of total lipides from animal tissues. J Biol Chem 226, 497-509.

23. Christie WW (2003) Lipid Analysis, 3rd ed. Oxford: The Oily Press/Pergamon Press.

24. Martinelli N, Girelli D, Malerba G, et al. (2008) FADS genotypes and desaturase activity estimated by the ratio of arachidonic acid to linoleic acid are associated with inflammation and coronary artery disease. Am J Clin Nutr 88, 941-949.

25. Bokor S, Dumont J, Spinneker A, et al. (2010) Single nucleotide polymorphisms in the FADS gene cluster are associated with delta- 5 and delta- 6 desaturase activities estimated by serum fatty acid ratios. J Lipid Res 51, 2325-2333.

26. Lemaitre RN, Tanaka T, Tang W, et al. (2011) Genetic loci associated with plasma phospholipid $n-3$ fatty acids: a meta-analysis of genome-wide association studies from the CHARGE consortium. PLoS Genet 7, e1002193.

27. Xie L \& Innis SM (2008) Genetic variants of the FADS1 FADS2 gene cluster are associated with altered ( $n-6)$ and (n-3) essential fatty acids in plasma and erythrocyte 
phospholipids in women during pregnancy and in breast milk during lactation. J Nutr 138, 2222-2228.

28. Baylin A, Ruiz-Narvaez E, Kraft P, et al. (2007) Alpha-linolenic acid, delta(6)-desaturase gene polymorphism, and the risk of nonfatal myocardial infarction. Am J Clin Nutr $\mathbf{8 5}$, 554-560.

29. Dunbar BL \& Bauer JE (2002) Conversion of essential fatty acids by Delta 6-desaturase in dog liver microsomes. $J$ Nutr 132, 1701S-1703S.

30. Francis DS, Peters DJ \& Turchini GM (2009) Apparent in vivo $\Delta$-6 desaturase activity, efficiency, and affinity are affected by total dietary C18 PUFA in the freshwater fish Murray cod. J Agric Food Chem 57, 4381-4390.

31. Thanuthong T, Francis DS, Senadheera SPSD, et al. (2011) LC-PUFA biosynthesis in rainbow trout is substrate limited: use of the whole body fatty acid balance method and different 18:3n-3/18:2n-6 ratios. Lipids 46, 1111-1127.
32. Ruxton C, Reed S, Simpson M, et al. (2007) The health benefits of omega- 3 polyunsaturated fatty acids: a review of the evidence. J Hum Nutr Diet 20, 275-285.

33. Stahl LA, Begg DP, Weisinger RS, et al. (2008) The role of omega-3 fatty acids in mood disorders. Curr Opin Investig Drugs 9, 57-64.

34. Wang C, Harris WS, Chung M, et al. (2006) n-3 Fatty acids from fish or fish-oil supplements, but not $\alpha$-linolenic acid, benefit cardiovascular disease outcomes in primary- and secondary-prevention studies: a systematic review. Am J Clin Nutr 84, 5-17.

35. Harris WS (2007) International recommendations for consumption of long-chain omega-3 fatty acids. $J$ Cardiovasc Med 8, S50-S52.

36. Turchini GM, Nichols PD, Barrow C, et al. (2012) Jumping on the omega-3 bandwagon: distinguishing the role of long-chain and short-chain omega-3 fatty acids. Crit Rev Food Sci Nutr 52, 795-803. 